\section{Measurement of Spinal Canal and Sagittal Balance of the Pelvis-Spine Complex in Nor- mal Congolese Population}

\section{Mukaya J*, Kabeya JM, Mbongo A and Mbuyi-Muamba JM}

Department of Radiology, University Hospital of Kinshasa, The Democratic Republic of the Congo

\begin{abstract}
Background

This study aimed to determine the measurement of spinal canal and spinopelvic parameters in Congolese population of Kinshasa.

Materials and methods

212 clinically asymptomatic were enrolled during 2014-2015; 138 subjects $(65.1 \%)$ were male and $74(34.9 \%)$ were female of matching age between 18 and 30 years.

Radiological study consisted of simple anteroposterior and lateral radiographs as well as computed tomography of the lumbar spine.

The following spinopelvic parameters were measured PI (Pelvic Incidence), Lumbar Lordosis (LL), Sacral Slope (SS) and Pelvic Tilt (PT).

Measurements in axial scan included midline Anteroposterior Vertebral Body diameter (APVB), midline Anteroposterior Canal Diameter (APCD), midline Dural Sac Diameter (DSD), Dural sac Cross-sectional Area (DCA), Canal Cross-Sectional Area (CCA), Interarticular Distance (IAD), Interpedicular Distance (IPD), Foraminal Height (FH) and Lateral Recess (LR). The Lumbar Ratio (LR) was calculated by dividing APCD by APVB.

Results

The mean \pm SD of the measurements at the narrowest level except L5-S1 was:

$\mathrm{APCD}=15.12 \pm 2.30 \mathrm{~mm}, \mathrm{IPD}=20.31 \pm 3.50 \mathrm{~mm}, \mathrm{LR}=5.35$ $\pm 1.40 \mathrm{~mm}, I A D=16.32 \pm 2.22 \mathrm{~mm}, D S D=12.12 \pm 2.11 \mathrm{~mm}$, $\mathrm{FH}=18.50 \pm 1.7 \mathrm{~mm}, \mathrm{DCA}=169.27 \pm 48.54 \mathrm{~mm} 2, \mathrm{CCA}=251.80 \pm$ $36.10 \mathrm{~mm}^{2}$ and $R=0.54 \pm 0.02$.

The mean \pm SD spinal and pelvic parameters were: $L L=61.1 \pm$ $9.7^{\circ}, \mathrm{PI}=54.4 \pm 7.8^{\circ}, \mathrm{SS}=41.0 \pm 8.4^{\circ} \mathrm{PV}=13.2 \pm 6.5^{\circ}$.
\end{abstract}

*Corresponding author: Mukaya J, Department of Radiology, University Hospital of Kinshasa, The Democratic Republic of the Congo, Tel: +243 998162458 ; E-mail: mtj@perso.be

Citation: Mukaya J, Kabeya JM, Mbongo A, Mbuyi-Muamba JM (2016) Measurement of Spinal Canal and Sagittal Balance of the Pelvis-Spine Complex in Normal Congolese Population. J Orthop Res Physiother 2: 025.

Received: June 21, 2016; Accepted: August 02, 2016; Published: August 16, 2016

\section{Conclusion}

The report is the first to present the normal radiological anatomy of different diameters of the lumbar spinal canal among adult Congolese population. These diameters are smaller than the measurement in Caucasian population.

Keywords: Congolese; Lumbar spinal canal; Measure; Spinopelvic parameter

\section{Introduction}

In the purpose of establishing normal dimension of the spinal canal, some authors have measured the spinal canal's diameter. That has been the case of Hinck et al., in Caucasians Latino America [1], Eisenstein et al., among black and white people in South Africa [2], Amonoo-in kuofi et al., among Nigerians [3], Orthan et al., among Turks [4], Ongolo et al., among Cameroon [5] and Nirvan et al., among India [6]. Racial and ethnic variations have been noted [7].

Few years ago, sagittal parameters of the spine have become inescapable and have constituted a revolution in the diagnostic and therapeutic of spinal disorders [8]. However, in Democratic Republic of Congo (DRC), the profile of the spinal canal and sagittal parameters has never been documented.

The purpose of this study is to determine the normal range of different diameters of canal and sagittal spinal in normal Congolese population.

\section{Materials and Methods}

\section{Subjects}

212 clinically normal adult males and females of matching age between 18 and 30 years were selected for the present study. The subjects were volunteers from various city of Kinshasa.

Exclusion criteria were: past or present back pain, presence of spinal diseases, spinal configuration anomalies, history of spinal traumatism, metabolic diseases, pregnancy.

\section{Collection of demographic and medical data}

At each selected topic, the demographics (age and sex) and clinical (height and weight) information were collected. Body Mass Index (BMI) was calculated as weight in kilograms ( $\mathrm{kg}$ ) divided per the square of height in meters $(\mathrm{m})$.

\section{Radiological study}

Radiological study consisted of simple anteroposterior and lateral radiographs in standing position as well as computed tomography of the lumbar spine. Special care was taken to visualize both femoral heads on this $\mathrm{x}$ ray. CT (Computed Tomography) scans performed with patients in the supine position using Somaton Sensation 64 (Siemens, Germany).

Spinopelvic parameters (Figure 1), the morphological type of the canal spinal, the spinal canal configuration and the different measures by a single observer. 


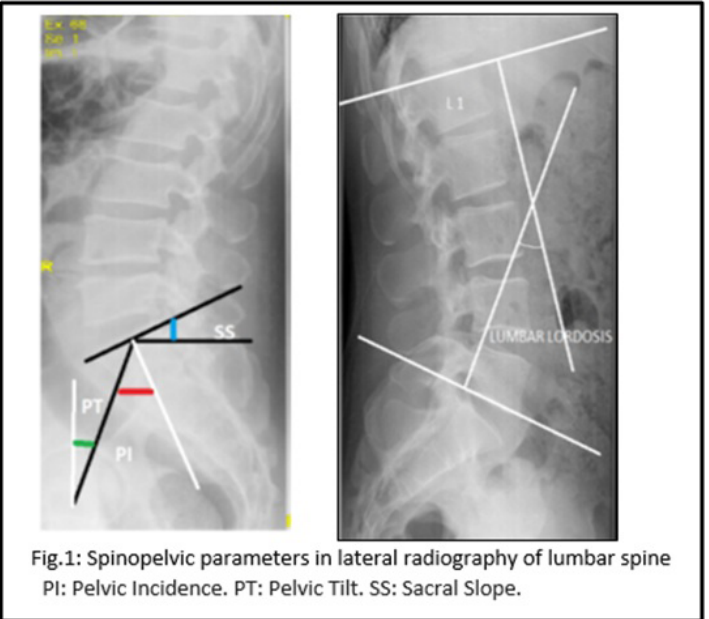

Figure 1: Spinopelvic parameters in lateral radiography of lumbar spine.

The following spinopelvic parameters were measured using the worldwide principal protocol [9]:

- PI (Pelvic Incidence) is measured as the angle between a line drawn perpendicular to the sacral end plate at its midpoint of the femoral head axis;

- Lumbar Lordosis (LL) is the sagittal Cobb angle measured between the superior end plate of L1 and the inferior end plate of L5;

- Sacral Slope (SS), angle between the horizontal and sacral plate;

- Pelvic Tilt (PT), angle between the vertical and the line through the midpoint of the sacral plate to femoral heads axis.

Most measurements in this study were based on those in published studies [9-11] in CT. Cuts below the pedicles were made through the highest part of the intervertebral foramina and the following parameters were measured (Figure 2): midline Anteroposterior Vertebral Body diameter (APVB), midline Anteroposterior Canal Diameter (APCD), Canal Cross-Sectional Area (CCA) and Interpedicular Distance (IPD). These cuts were made at the level of the middle of disc and are concerned with the intervertebral articulation and the following parameters were measured: midline Dural Sac Diameter (DSD), Dural sac Cross-sectional Area (DCA) and Interarticular Distance (IAD). The others cuts were made to measure Foraminal Height (FH), the Lateral Recess (LR).

The lumbar Ratio (R) was calculated by dividing APCD by APVB. Likewise, measurements that were less than the mean - 2 SD were considered to be "below normal".

\section{Reproducibility}

106 patients were randomly selected for evaluation of inter observer variability of measurement by two independent observers. To obtain intra observer variability, the same measurement was repeater after 1 month. The intra and inter observer reliability was excellent $(\mathrm{Kappa}=0.92)$ and $(\mathrm{Kappa}=0.64)$ right respectively.

\section{Statistical analysis}

Statistical analysis was performed using SPSS 20.0 for windows (SPSS Inc. Chicago, IL, USA).

Data are expressed as percentages and the mean \pm standard deviation. The relationship between two continuous variables was assessed using a bivariate correlation method (Pearson's correlation).

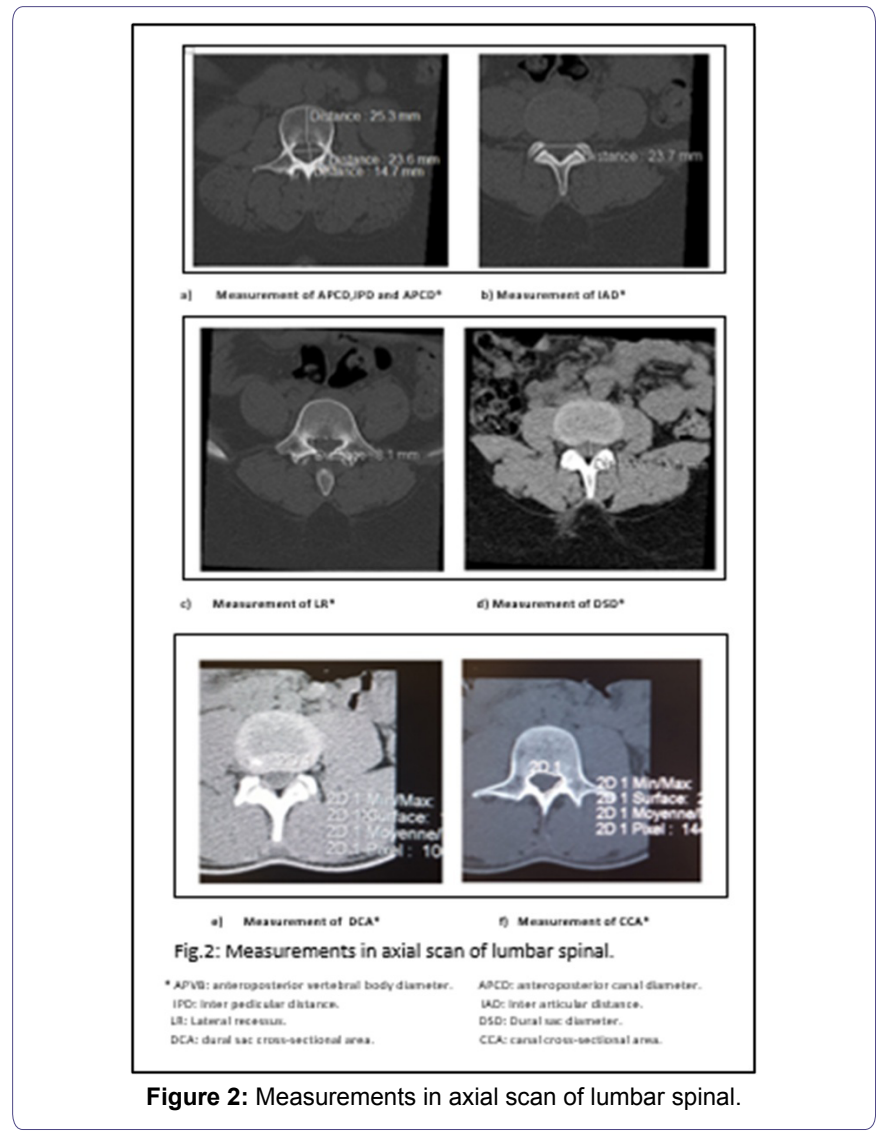

\section{Results}

\section{Description of the study population}

The study population consisted of 212 subjects with a median age of $24.2 \pm 2.2$ years old ( 74 female's and 138 male's). Weight, height and BMI means of participants were $56.8 \mathrm{~kg}$, respectively, $167.3 \mathrm{~cm}$ and $20.5 \mathrm{~kg} / \mathrm{m}^{2}$. Three out of $10(29.7 \%)$ were lean $\left(\mathrm{BMI}<18.5 \mathrm{~kg} / \mathrm{m}^{2}\right)$, overweight and obesity were however $8.5 \%$ and $1.4 \%$ respectively (Table 1).

\begin{tabular}{|c|c|}
\hline Variable & Patients \\
\hline Sociodemographic data & \\
\hline Age & Mean SD $24.2 \pm 2.2$ years \\
\hline Gender & $74(34.9 \%)$ \\
\hline Female & $138(65.1 \%)$ \\
\hline Male & \\
\hline Anthropometric data & Mean SD $56.8 \pm 7.4$ \\
\hline Weight (Kg) & Mean SD $167.3 \pm 9.7$ \\
\hline Height (Centimeter) & Mean SD $20.5 \pm 3.7$ \\
\hline BMI (Kg/m $\left.{ }^{2}\right)$ & $63(29.7 \%)$ \\
\hline$<18.5$ & $128(60.4 \%)$ \\
\hline $18.5-24.5$ & $3(1.4 \%)$ \\
\hline$\geq 30$ &
\end{tabular}

Table 1: Patient Sociodemographic and Anthropometric.

\section{Quantitative measurements}

The mean \pm SD of the measurements at the narrowest level except L5-S1 and the minimum dimension are summarized in table 2. The 
normal value are: $\mathrm{APCD} \geq 10.5 \mathrm{~mm}, \mathrm{IPD} \geq 16 \mathrm{~mm}, \mathrm{LR} \geq 2.5 \mathrm{~mm}$ IAD $\geq 11.5 \mathrm{~mm}$, DSD $\geq 7.5$, DCA $\geq 70 \mathrm{~mm}^{2}$, CCA $\geq 179 \mathrm{~mm}^{2}$ and $\mathrm{TR} \geq 0.50$.

\begin{tabular}{|c|c|c|}
\hline Parameter & Mean \pm SD & Minimum measurement \\
\hline APCD $(\mathrm{mm})$ & $15.12 \pm 2.30$ & 10.5 \\
\hline IPD $(\mathrm{mm})$ & $20.31 \pm 3.50$ & 16 \\
\hline LR $(\mathrm{mm})$ & $5.35 \pm 1.40$ & 2.5 \\
\hline IAD $(\mathrm{mm})$ & $16.32 \pm 2.22$ & 11.5 \\
\hline DSD $(\mathrm{mm})$ & $12.12 \pm 2.11$ & 7.5 \\
\hline FH $(\mathrm{mm})$ & $18.50 \pm 1.7$ & 15 \\
\hline DCA $\left(\mathrm{mm}{ }^{2}\right)$ & $169.27 \pm 48.54$ & 70 \\
\hline CCA $\left(\mathrm{mm}^{2}\right)$ & $251.80 \pm 36.10$ & 179 \\
\hline$R$ & $0.54 \pm 0.02$ & 0.5 \\
\hline
\end{tabular}

Table 2: Average dimensions of lumbar spinal canals in 212 asymptomatic volunteers at the narrowest level except L5-S1 and minimum measurement. APCD: Anteroposterior Canal Diameter; CCA: Canal Cross-Sectional Area; DCA: Dural sac Cross-sectional Area; DSD: Dural Sac Diameter; FH: Foraminal Height; IAD: Inter Articular Diameter; IPD: Inter Pedicular Diameter; LR: Lateral Recessus; R: Lumbar Ratio

Spinal and pelvic parameters of the sagittal balance of the pelvis-spine complex

The mean \pm SD minimum and maximum spinal and pelvic parameters are summarized in table 3 . The average lordosis was $61.1 \pm 9.7^{\circ}$, pelvic incidence was $54.4 \pm 7.8^{\circ}, 41.0$ a sacral slope of $\pm 8.4^{\circ}$ and pelvic version $13.2 \pm 6.5^{\circ}$

\begin{tabular}{|c|c|c|c|}
\hline Parameter & Mean SD & Minimum & Maximum \\
\hline Lumbar lordosis $\left({ }^{\circ}\right)$ & $61.1 \pm 9.7$ & 40.8 & 84.1 \\
\hline Pelvic incidence $\left({ }^{\circ}\right)$ & $54.4 \pm 7.8$ & 31.2 & 79.5 \\
\hline Sacral slope $\left({ }^{\circ}\right)$ & $41.0 \pm 8.4$ & 0.2 & 56.6 \\
\hline Pelvic tilt $\left(^{\circ}\right)$ & $13.2 \pm 6.5$ & 6.4 & 34.1 \\
\hline
\end{tabular}

Table 3: Pelvic and spinal parameters of sagittal balance.

There was a close correlation between sacral slope and pelvic incidence $(r=0.61)$, lumbar lordosis and sacral slope $(r=0.64)$, lumbar lordosis and pelvic incidence $(r=0.68)$ (Table 4$)$.

\begin{tabular}{|c|c|c|c|c|}
\hline Parameter & PI & SS & PT & LL \\
\hline PI & 1 & - & - & - \\
\hline SS & 0.61 & 1 & - & - \\
\hline & $<0.001$ & & & - \\
\hline PT & 0.02 & -0.18 & 1 & - \\
\hline & $<0.828$ & 0.208 & & -0.34 \\
\hline LL & 0.68 & 0.64 & 0.17 & \\
\hline & $<0.001$ & $<0.001$ & & \\
\hline
\end{tabular}

Table 4: Correlation matrix among the spinal and pelvic parameters. Upper line: Correlation coefficient; Lower line: $p$-values

LL: Lumbar Lordotic; PI: Pelvic Incidence; PT: Pelvic Tilt; SS: Sacral Slope

\section{Discussion}

The average age of the study population was 24.2 years of which $65.1 \%$ were male against $34.9 \%$ female with an average size of $167.3 \mathrm{~cm}$, an average weight of 56.8 and a BMI of $20.5 \mathrm{~kg} / \mathrm{cm}$ (Table 1). The male dominance can be explained in this random sample that by easy acceptability of men at risk and exclusion of women in cases of amenorrhea, or the date of the last unknown rules.

The youth of our sample was justified by the fact that bone growth is usually completed at the age of 17 years and maximum of 30 years in that before this age the risk of bone degeneration is low.

The main result of this research was to determine reference values of lumbar spinal canals. The threshold values defining the anatomical lumbar stenosis were: DAP $<10.5 \mathrm{~mm} \mathrm{DIP}<16 \mathrm{~mm}, \mathrm{LP}<2.5 \mathrm{~mm}$, DIA $<11.5 \mathrm{~mm}, \mathrm{DFD}<7.5 \mathrm{~mm}, \mathrm{DF}<15 \mathrm{~mm}, \mathrm{C} / \mathrm{V}<0.50, \mathrm{SC}<179$ $\mathrm{mm}^{2}$ and $\mathrm{SD}<70 \mathrm{~mm}^{2}$

These values are well below the values proposed by the classical treatises. Indeed, for classical treatises of French literature, DAP has values:

- Normal value $\geq 15 \mathrm{~mm}$

- Closeness on: 13 and $14 \mathrm{~mm}$

- Closeness absolute $\leq 12 \mathrm{~mm}$ [10-15].

For the DFD, cited the normal dimensions of at least $11 \mathrm{~mm}$ [10-15].

In English literature, the "Atlas of radiology measurement" by Keats TE and Silstrom C. Mosby 2001 [16] cites as normal value $\pm 16 \mathrm{~mm} 3$ in L4 of DAP.

These reference values are no less indicative as severe strictures are not incompatible with the absence of symptoms and, conversely, even normal dimensions on supine exams can be significantly reduced by the static conditions or dynamics of standing. In addition, the statistical standard does not necessarily correspond to the boundary between normality and pathology: an individual whose actions deviate more than two standard leads do not mean a sick individual.

Analysis of the sagittal balance of the spine is a fundamental step in the understanding and treatment of many spinal pathologies. One objective of this work was to determine the physiological values of pelvic and spinal parameters of sagittal balance of the spine and to study their interrelations. Hyperlordosis of African described by Courson in 1963 [16] before a clinical aspect of high-lumbar curve corresponds to different values of the pelvic and spinal sagittal balance parameters of the lumbar spine?

The average values of the main angular parameters considered were: lumbar lordosis 61.1 degrees $( \pm 9.7)$, sacral slope 41.0 degrees $( \pm 8.4$ ), pelvic Version 13.2 degrees $( \pm 6.5)$ and 54.4 degrees pelvic incidence $( \pm 7.8)$. There was a close correlation between sacral slope and pelvic incidence $(r=0.61)$, lumbar lordosis and sacral slope $(r=0.64)$, lumbar lordosis and pelvic incidence $(r=0.68)$.

Morphotype pelvic spinal black Africans present values comparable to the literature [8] and meets the same provisions of anatomical and functional interdependence of sagittal balance of the lumbar spine.

\section{Conclusion}

The report is the first to present the normal radiological anatomy of different diameters of the lumbar spinal canal among adult Congolese population. These diameters are smaller than the measurement in Caucasian population.

\section{Ethics and Disclosures}

The protocol for the study was approved by the institutional review board of Kinshasa university hospital (ESP/CE/009/2016). Informed 
consents have been obtained in writing from patients, and all clinical investigations were conducted according to the principles expressed in the declaration of Helsinki. The patients have given their consent for publications of the clinical details.

There was no funding received for this study and there was no conflict of interests.

\section{References}

1. Hinck VC, Clack WM Jr, Hopkins CE (1966) Normal interpediculate distances (minimum and maximum) in children and adults. Am J Roentgenol Radium Ther Nucl Med 97: 141-153.

2. Eisenstein S (1977) The morphometry and pathological anatomy of the lumbar spine in South African negroes and caucasoids with specific reference to spinal stenosis. J Bone Joint Surg Br 59: 173-180.

3. Amonoo-Kuofi HS (1985) The sagittal diameter of the lumbar vertebral canal in normal adult Nigerians. J Anat 140: 69-78.

4. Tacar O, Demirant A, Nas K, Altindağ O (2003) Morphology of the lumbar spinal canal in normal adult Turks. Yonsei Med J 44: 679-685.

5. Zogo OP, Minkala LTM, Juimo AG (2011) Biometrie scanographique du canal rachidien lombaire de l'adulte camerounais. Journal Africain d'Imagerie Médicale 3: 308-317.

6. Nirvan AB, Pensi CA, Patel JP, Shah GV, Dave RV (2005) A study of inter-pedicular distances of the lumbar vertebrae measured in plain antero-posterior radiograph in Gujaratis. J Anat Soc. India 54: 1-9.

7. Karantanas AH, Zibis AH, Papaliaga M, Georgiou E, Rousogiannis S (1998) Dimensions of the lumbar spinal canal: variations and correlations with somatometric parameters using C.T. Eur Radiol 8: 1581-1585.
8. Mac-Thiong JM, Roussouly P, Berthonnaud E, Guigui P (2010) Sagittal parameters of global spinal balance: normative values from a prospective cohort of seven hundred nine Caucasian asymptomatic adults. Spine (Phila Pa 1976) 35: 1193-1198.

9. Legaye J, Duval-Beaupère G, Hecquet J, Marty C (1998) Pelvic incidence: a fundamental pelvic parameter for three-dimensional regulation of spinal sagittal curves. Eur Spine J 7: 99-103.

10. Mamisch N, Brumann M, Hodler J, Held U, Brunner F, et al. (2012) Radiologic criteria for the diagnosis of spinal stenosis: results of a Delphi Survey. Radiology 1: 174-179.

11. Ulrich CG, Binet EF, Sanecki MG, Kieffer SA (1980) Quantitative assessment of the lumbar spinal canal by computed tomography. Radiology 134: 137-143.

12. Laredo JD, Abi-ayad A (1998) Canaux lombaires centraux étroits et rétrécis. In: Morvan G, Laredo JD, Wybier M (eds.). Imagerie ostéo-articulaire. Flammarion, Paris, France.

13. Chevrot A, Beges C, Drapé JL (1998) Conduite de l'imagerie dans les sténoses lombaires. In: Morvan G, Deburge A, Bard H, Laredo JD (eds.). Le rachis lombaire dégénératif. GETROA Opus XXV, Sauramps Medical, Montpellier, France. Pg no: 373-389.

14. Dietemann JL (1995) Sténoses du canal rachidien lombaire. In Imagerie du rachis lombaire. Masson, Paris. 78-92.

15. Cotton A (2008) Imagerie musculosquelettique: Pathologies locorégionales Elsevier Masson.

16. Keats TE, Sistrom C (2001) Measurement of the sagittal diameter of the lumbar spinal canal in children and adults. Atlas of radiologic measurement, Mosby, Missouri, USA. 